\section{In the news}

\section{FROM THE EACR-ESMO LIQUID BIOPSY MEETING}

Reflecting the rapidly increasing level of research and clinical interest in liquid biopsies, May 2019 saw the first EACR-ESMO Joint Conference on Liquid Biopsies, in Bergamo, Italy.

In the opening address, Nick Papadopoulos described the possibility of achieving the ultimate goal, or 'holy grail' of liquid biopsy research - the use of liquid biopsies for routine screening and early detection of cancers - and this ultimate goal emerged as a common theme of the meeting. Opinions vary as to how feasible an objective this is, although a general agreement emerged among delegates regarding the need to combine measures of several different circulating biomarkers, thus emphasizing the importance of integrating research involving circulating tumour cells, epigenetic alterations, RNAs, exosomes and other circulating material, in addition to circulating tumour DNA (ctDNA), all of which were featured in this meeting.

The enormous clinical potential of liquid biopsy-based assays was discussed in detail, including sessions devoted to monitoring of tumour evolution and minimal residual disease during and after treatment. Here, emerging data from use of ctDNA-based assays to monitor acquired resistance in patients with colorectal cancer or non-small-cell lung cancer are providing early examples of the clinical implementation of this technology.

The lively panel debate on the future of liquid biopsy research was another notable highlight. Many themes emerged from this debate, including the currently limited financial incentives for the development of a circulating biomarker that might then restrict the use of a novel anticancer drug to a subpopulation of patients.

A final observation, supported by virtually all the speakers, is that many questions relating to the biology of circulating tumour material remain unanswered. The need for robust, reproducible basic science must not be overlooked in the drive towards the development of clinical assays. Indeed, such research will be essential to the many attempts to harness liquid biopsy-based technologies.

Closing the meeting, EACR president Alberto Bardelli noted that, owing to the high level of interest shown, further meetings focusing on this area of research are likely to follow shortly.

Peter Sidaway

\title{
Benefit from LCT in oligometastatic NSCLC
}

Retrospective or single-arm prospective studies have shown that local consolidative therapy (LCT) can extend progression-free survival (PFS) in patients with oligometastatic disease. An overall survival (OS) benefit in patients with non-small-cell lung cancer (NSCLC) has now been demonstrated in a prospective randomized phase II trial.

Patients with advanced-stage NSCLC and $\leq 3$ metastatic lesions without progression $\geq 3$ months after front-line systemic therapy received standard maintenance therapy or observation with $(n=25)$ or without $(n=24)$ LCT (with surgery and/or radiotherapy).

At a median of 38.8 months, LCT was associated with an OS benefit: median 41.2 months versus 17.0 months $(P=0.017)$. Previously published results of this trial indicated a statistically significant PFS benefit with LCT that has now been confirmed: 14.2 months versus 4.4 months $(P=0.022)$.

OS durations after disease progression were longer in the LCT arm than in the control arm: median 37.6 months versus 9.4 months $(P=0.034)$. Salvage therapy for these patients involved additional systemic agents and/or LCT to all progressing sites. Patients who received LCT-based salvage therapy (41\%) had longer OS durations than those who received other salvage therapies: median not reached (NR; 95\% CI 11.5 months-NR) versus 16.4 months (95\% CI 8.7-40.9 months; $P=0.01$ ). The previously reported incidence of grade $\geq 3$ toxicities was $20 \%$ in the LCT arm versus $8 \%$ in the control arm; no additional events were described.

Together with the recently published results of the SABR-COMET trial, this study contributes to the growing evidence of a curative role of local ablative therapies in patients with oligometastatic cancer.

Diana Romero

ORIGINAL ARTICLE Gomez, D. R. et al. Local consolidative therapy vs. maintenance therapy or observation for patients with oligometastatic non-small-cell lung cancer: long-term results of a multi-institutional, phase II, randomized study. J.Clin. Oncol.https://doi.org/10.1200/JCO.19.00201 (2019) FURTHER READING Gomez, D. R. et al. Local consolidative therapy versus maintenance therapy or observation for patients with oligometastatic non-small-cell lung cancer without progression after first-line systemic therapy: a multicentre, randomised, controlled, phase 2 study. Lancet Oncol. 17, 1672-1682 (2016)

BREAST CANCER

\section{Alpelisib effective in advanced- stage disease}

The majority of women with hormone receptor $(\mathrm{HR})^{+}, \mathrm{HER} 2^{-}$breast cancer respond to endocrine therapy, although acquired resistance is common. Now, data from a phase III trial demonstrate the efficacy of the potent $\alpha$-isoform-specific phosphatidylinositol 3-kinase- $\alpha$ (PI3Ka) inhibitor alpelisib.

In this international, multicentre trial, a cohort of 572 patients with locally confirmed $\mathrm{HR}^{+}$, HER2 ${ }^{-}$advanced-stage breast cancer, of whom 341 had confirmed PIK3CA alterations, were randomized $(1: 1)$ to receive alpelisib plus fulvestrant or placebo plus fulvestrant. Progression-free survival (PFS) specifically in patients with PIK3CA-mutant disease was the primary end point.

After a median follow-up duration of 20 months, patients in the alpelisib group with PIK3CA-mutant disease had a median PFS of 11.0 months versus 5.7 months in the placebo group (hazard ratio for disease progression or death $0.65,95 \% \mathrm{Cl} 0.50-0.85 ; \mathrm{P}<0.001)$. This difference was not observed in comparisons of the outcomes of patients with PIK3CA-wild-type disease (median PFS 7.4 versus 5.6 months respectively, hazard ratio $0.85,95 \% \mathrm{Cl} 0.58-1.25$ ).

Grade 3 or 4 adverse events were observed in $64.4 \%$ and $11.6 \%$ of patients in the alpelisib group, versus $30.3 \%$ and $5.2 \%$ among those receiving placebo, resulting in treatment discontinuations in $25 \%$ versus $4.2 \%$ of patients, respectively. Among these events, grade 3 hyperglycaemia (including diabetes mellitus, insulin resistance and metabolic syndrome) was observed in $32.7 \%$ of patients receiving alpelisib.

These findings confirm the feasibility and efficacy of targeting PI3Ka using alpelisib in the approximately $40 \%$ of patients with PIK3CAmutant advanced-stage $\mathrm{HR}^{+}$, HER2- breast cancer and confirm the role of PIK3CA mutations as a biomarker. The high risk of hyperglycaemia-related adverse events, which the investigators considered to be an on-target effect, indicates the need for close on-treatment monitoring.

Peter Sidaway

ORIGINAL ARTICLE André, F. et al. Alpelisib for PIK3CA-

mutated, hormone receptor-positive advanced breast cancer. N. Engl.J. Med. 380, 1929-1940 (2019) 